\title{
Decreasing CNPY2 Expression Diminishes Colorectal Tumor Growth and Development through Activation of p53 Pathway
}

Ping Yan, ${ }^{*}$ Hui Gong, ${ }^{*}$ Xiaoyan Zhai, ${ }^{*}$ Yi Feng, ${ }^{* \dagger}$ Jun Wu, ${ }^{\ddagger}$ Sheng He, ${ }^{*}$ Jian Guo, ${ }^{\ddagger}$ Xiaoxia Wang, ${ }^{*}$ Rui Guo, ${ }^{*}$ Jun Xie, ${ }^{*}$ and Ren-Ke Li

\begin{abstract}
From the Department of Biochemistry and Molecular Biology,* Ministry of Education Key Laboratory of Cellular Physiology, Shanxi Medical University, Taiyuan, China; the Department of Colorectal Surgery, ${ }^{\dagger}$ Shanxi Cancer Hospital, Taiyuan, China; and the Division of Cardiovascular Surgery, ${ }^{\ddagger}$ Toronto General Research Institute, University Health Network, Toronto, Ontario, Canada
\end{abstract}

\author{
Accepted for publication \\ November 19, 2015. \\ Address correspondence to \\ Jun Xie, Ph.D., Department of \\ Biochemistry and Molecular \\ Biology, Ministry of Educa- \\ tion Key Laboratory of \\ Cellular Physiology, Shanxi \\ Medical University, Taiyuan \\ 030001, China; or Ren-Ke Li, \\ M.D., Ph.D., Toronto General \\ Research Institute, MaRS \\ Centre, Toronto Medical Dis- \\ covery Tower, Room 3-702, \\ 101 College St, Toronto, ON, \\ Canada M5G 1L7. E-mail: \\ xiejun1968@126.com or \\ renkeli@uhnres.utoronto.ca.
}

\begin{abstract}
Neovascularization drives tumor development, and angiogenic factors are important neovascularization initiators. We recently identified the secreted angiogenic factor CNPY2, but its involvement in cancer has not been explored. Herein, we investigate CNPY2's role in human colorectal cancer (CRC) development. Tumor samples were obtained from CRC patients undergoing surgery. Canopy 2 (CNPY2) expression was analyzed in tumor and adjacent normal tissue. Stable lines of human HCT116 cells expressing CNPY2 shRNA or control shRNA were established. To determine CNPY2's effects on tumor xenografts in vivo, human CNPY2 shRNA HCT116 cells and controls were injected into nude mice, separately. Cellular apoptosis, growth, and angiogenesis in the xenografts were evaluated. CNPY2 expression was significantly higher in CRC tissues. CNPY2 knockdown in HCT116 cells inhibited growth and migration and promoted apoptosis. In xenografts, CNPY2 knockdown prevented tumor growth and angiogenesis and promoted apoptosis. Knockdown of CNPY2 in the HCT116 CRC cell line reversibly increased p53 activity. The p53 activation increased cyclin-dependent kinase inhibitor p21 and decreased cyclin-dependent kinase 2, thereby inhibiting tumor cell growth, inducing cell apoptosis, and reducing angiogenesis both in vitro and in vivo. CNPY2 may play a critical role in CRC development by enhancing cell proliferation, migration, and angiogenesis and by inhibiting apoptosis through negative regulation of the p53 pathway. Therefore, CNPY2 may represent a novel CRC therapeutic target and prognostic indicator. (Am J Pathol 2016, 186: 1015-1024; http://dx.doi.org/10.1016/j.ajpath.2015.11.012)
\end{abstract}

Despite effective surgical procedures and adjuvant chemotherapy for colorectal cancer (CRC) patients, approximately $50 \%$ of them initially thought to be cured by surgery subsequently relapse and die of their disease. ${ }^{1}$ Annual projections indicated that 1,300,000 individuals would be diagnosed with CRC last year and 600,000 patients would die from it. ${ }^{2}$ Much remains to be determined about the pathogenesis of CRC. Exploration of the etiology of CRC for early prevention and diagnosis and to facilitate the development of new therapeutic strategies is urgently needed.

Cancer growth and development are dependent on angiogenesis, and neovascularization has been implicated in the progression of many malignancies, including CRC. ${ }^{3}$
Investigating angiogenesis in tumor tissue increases our understanding of cancer biology and aids development of new therapeutic approaches. Neovascularization is a complex biological process, and vessel formation requires coordinated cellular action stimulated by angiogenic growth factors, which initiate blood vessel formation and

\footnotetext{
Supported by the Shanxi Scholarship Council of China (2013-55), the Shanxi Medical University Youth Research Fund grants Q02201201 and Q02201210, and partially by the Heart and Stroke Foundation of Canada grant G15009023. R.K.L. holds a Canada Research Chair in Cardiac Regeneration.

P.Y. and H.G. contributed equally to this work.

Disclosures: None declared.
} 
determine the type of vessels (capillaries or arterioles) that are formed. Angiogenesis begins when the function of pro-angiogenic activators outweighs that of angiogenic inhibitors. Several studies have demonstrated the importance of pro-angiogenic vascular endothelial growth factor (VEGF) in mediating tumor angiogenesis and growth. ${ }^{4}$ These studies have hypothesized that CRC cells up-regulate VEGF, thereby activating the process of neovascularization.

Pro-angiogenic genes are often up-regulated during hypoxia. In a screen of genes encoding predicted secreted factors whose expression is increased during hypoxia in human smooth muscle cells, we identified Canopy 2 (CNPY2) as a potential angiogenic factor. Subsequently, we demonstrated that the CNPY2 transcript is controlled by hypoxia-inducible factor $1 \alpha(\mathrm{HIF}-1 \alpha)$, and CNPY2 protein is secreted from cells and can promote angiogenesis in vitro and in vivo. ${ }^{6}$ It has also been reported that elevated expression of tumor suppressor p53 inhibits HIF- $1 \alpha$, resulting in diminished expression of various HIF- $1 \alpha$-controlled angiogenic growth factors, which adversely affect systolic cardiac function. ${ }^{7}$ Because p53 has anti-cancer effects, ${ }^{8}$ there could be cross talk between p53 and CNPY2. In the current study, we hypothesized that the angiogenic factor CNPY2 may be involved in the growth and development of CRC through activation of the p53 pathway. We examined the expression of CNPY2 in primary CRCs and in patient plasma. To explore the influence of CNPY2 on CRC cell growth, migration, angiogenesis, and apoptosis, we established a stable HCT116 CRC cell line that constitutively expresses shRNA against CNPY2 to investigate the effect of CNPY2 knockdown on tumor cell biology. Furthermore, the p53 pathway was investigated to explore a possible association with $C N P Y 2$ affecting tumor cell growth and development. Finally, the effects of CNPY2 on tumor growth, angiogenesis, and apoptosis as well as the interaction with the p53 pathway were investigated using an in vivo xenograft tumor model.

\section{Materials and Methods}

\section{Ethics, Consent, and Permissions}

All human study protocols were approved by the ethics board of the Shanxi Medical University (Taiyuan, China) and conformed to the principles of the Declaration of Helsinki. All patients provided written informed consent.

All animal experiments were approved by the University of Shanxi Medical Animal Care and Use Committee.

\section{Tissue and Plasma Collection}

Tissue was collected from 28 patients diagnosed with CRC and undergoing surgery at the Shanxi Cancer Hospital. CRC and paracarcinomic normal tissues were harvested and either stored at $-80^{\circ} \mathrm{C}$ for biochemical studies or fixed and embedded in paraffin for immunohistochemistry. Blood plasma was collected from CRC patients $(n=19)$ and normal controls $(n=11)$.

\section{Establishment of Stably Transfected shRNA HCT116 Cell Lines}

The human HCT116 CRC cell line was obtained from the Shanxi Medical University Cell Bank. Cells were maintained in Dulbecco's modified Eagle's medium-high glucose (Life Technologies, Carlsbad, CA) containing $10 \%$ fetal bovine serum (Hyclone, South Logan, UT). HCT116 cells were transfected with $p R F P-C-R S$ containing shRNA against CNPY2 or a negative control shRNA (OriGene, Beijing, China) using Lipo2000 (Life Technologies). After 2 days, $0.5 \mu \mathrm{g} / \mathrm{mL}$ puromycin (Sigma-Aldrich, St. Louis, MO) was added to the culture medium, and cells were incubated for a further 21 days. Single transfected cells were selected by limited dilution. Cells were further cultured and subcultured to establish HCT116 shRNA-CNPY2 $_{\text {and }}$ HCT116 $6_{\text {shRNA-control }}$ stable transfected cell lines. A flow cytometric analysis was performed to verify transfection efficiency [\% of red fluorescent protein (RFP) ${ }^{+}$cells] using an FC500 flow cytometer (Beckman Coulter, Brea, CA).

\section{RT-PCR}

Total RNA was extracted using Trizol (Life Technologies). RNA $(2 \mu \mathrm{g})$ was reverse transcribed (Thermo Scientific, Waltham, MA), and $100 \mathrm{ng}$ of the resultant cDNA was PCR amplified (Thermo Scientific). The following primers were used: CNPY2, 5'-GAGGAGCCAGGATCTCCACT-3' (forward) and $5^{\prime}$-TCATCATGCGATATGTGCAG-3' (reverse); and $\beta$-actin, $5^{\prime}$-CCTGAAGTACCCCATCGAGC- $3^{\prime}$ (forward) and $5^{\prime}$-CTCTTGCTCGAAGTCCAGGG-3' (reverse). To exclude non-specific (DNA template) amplification, a negative control was introduced using a DNA template from rat heart tissue, which was different from human samples. For this purpose, total RNA was extracted from rat heart tissue and cDNA was synthesized by reverse transcription. A negative control PCR was performed using cDNA from rat heart tissue as a template and human $C N P Y 2$ specific primers with the conditions listed above. RT-PCR products were separated by gel electrophoresis, and image analysis was performed using ImageJ software version 1.49 (NIH, Bethesda, MD; http:// imagej.nih.gov/ij). Band brightness was quantified, and relative levels of CNPY2 mRNA were presented as the ratio of CNPY2 brightness/ $\beta$-actin brightness $(n=19$ for patient carcinomic and adjacent normal tissue pairs, $n=9$ replicates for HCT116 $6_{\text {shRNA-CNPY2 }}$ and HCT116 $6_{\text {shRNA-control }}$ cell lines).

\section{Western Blot Analysis and Enzyme-Linked Immunosorbent Assay}

Proteins were extracted in protein lysis buffer $[0.05 \mathrm{~mol} / \mathrm{L}$ Tris (pH 7.5), $0.5 \%$ sodium deoxycholate, and $0.1 \%$ SDS] with protease inhibitor cocktail (EMD Millipore, Billerica, MA). 
Table 1 Patient Characteristics

\begin{tabular}{llc}
\hline Sex & No. of patients & Age, years \\
\hline Male & 11 & $65.4 \pm 12.02$ \\
Female & 17 & $53.8 \pm 9.21$ \\
Total & 28 & $57.92 \pm 11.57$ \\
\hline
\end{tabular}

Data given as means \pm SD.

Protein concentrations were measured using a BCA assay kit (Pierce Biotechnology, Rockford, IL). Total protein $(40 \mu \mathrm{g})$ was separated by SDS-PAGE (100 V, 90 minutes), transferred to a polyvinylidene difluoride membrane, and blocked in 5\% nonfat milk before incubation with primary antibodies. The primary antibodies were as follows: CNPY2 (1:1000; Novus Biologicals, Littleton, CO); p53 and phospho-p53, cyclindependent kinase inhibitor $\mathrm{p} 21^{\text {Waf1 }}$ (p21), and cyclin-dependent kinase 2 (CDK2; all 1:1000; Cell Signaling Technology, Danvers, MA); glyceraldehyde-3-phosphate dehydrogenase (1:1000; Zhongshan Jinqiao, Beijing, China); $\beta$-actin (1:2000; Zhongshan Jinqiao) $(n=28$ for patient carcinomic and adjacent normal tissue pairs, $n=9$ replicates for HCT116 $6_{\text {shRNA-CNPY2 }}$ and HCT116 $6_{\text {shRNA-control }}$ cell lines for CNPY2, $n=3$ replicates for in vitro $\mathrm{p} 53$ and phosphop53, p21, CDK2 replicates for HCT116 $6_{\text {shRNA-CNPY2 }}$ and HCT116 $6_{\text {shRNA-control }}$ cell lines, $n=8$ replicates for in vivo CNPY2, p53, and phospho-p53, p21, and CDK2 for the shRNA-control and shRNA-CNPY2 knockdown cell line-induced xenograft tumor tissues).

CNPY2 protein expression was measured in patient plasma and conditioned media by enzyme-linked immunosorbent assay, according to the manufacturer's instructions (USCN Life Science, Wuhan, China; CRC patients, $n=19$; healthy controls, $n=11 ; n=6$ replicates for HCT116 $6_{\text {shRNA-CNPY2 }}$ and HCT116 $6_{\text {shRNA-control }}$ supernatants).

\section{Cell Growth Assay}

Cells were counted using the CCK-8 assay kit (Dojindo Laboratories, Kumamoto, Japan; $n=6$ ). One thousand cells per well were seeded in 96-well plates and treated with CCK-8 on days $0,1,3$, and 5. Absorbance was determined at $450 \mathrm{~nm}$, and cell growth curves expressed as the ratio of OD at day of interest: OD at day 0 . To distinguish viable versus nonviable cells, $2.4 \times 10^{4}$ cells per well were seeded in 12-well plates. Cells were counted on days 1, 2, 3, and 5 using a Bio-Rad TC20 Automated Cell Counter (Hercules, $\mathrm{CA} ; n=4$ ), which differentiated live cells from dead cells.

\section{Scratch Wound Cell Migration Assay}

Cells were seeded in 6-well plates, grown to $70 \%$ to $80 \%$ confluence, and a scratch wound was made through the middle of the wells using a pipette tip. Images were taken using an inverted microscope (Olympus, Tokyo, Japan). Scratch wound width was measured and analyzed by ImageJ software ( $n=5$ replicates).

\section{Colony Formation Assay}

Cells $\left(2 \times 10^{4}\right)$ were cultured in a 100 -mm dish, as described above. After 14 days, the numbers of colonies per $\mathrm{cm}^{2}$ were analyzed using ImageJ software ( $n=5$ replicates). For the three-dimensional soft agar colony growth, $3 \mathrm{~mL}$ Dulbecco's modified Eagle's medium-high glucose $(10 \%$ fetal bovine serum) containing $0.6 \%$ soft agar was added into a $60-\mathrm{mm}$ dish. After the soft agar was solidified, $2 \mathrm{~mL}$ Dulbecco's modified Eagle's medium (10\% fetal bovine serum) containing $0.3 \%$ soft agar and 2000 cells was laid on top. After 10
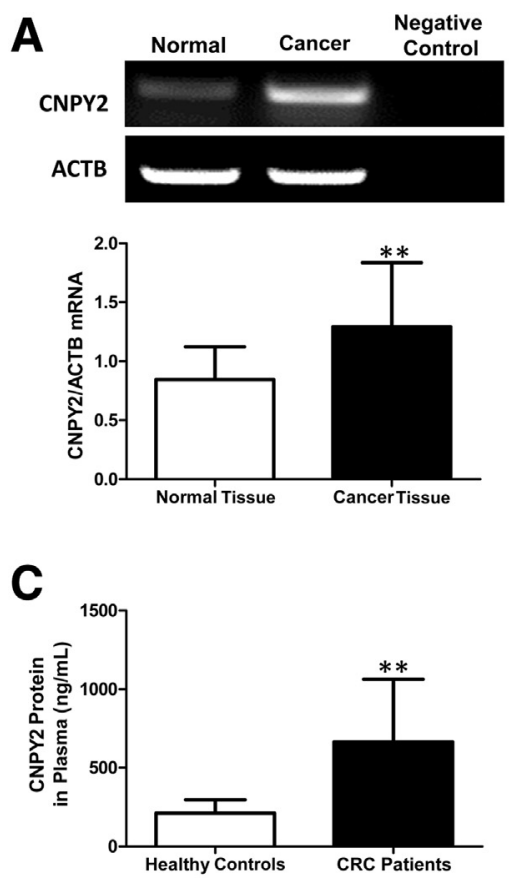

B
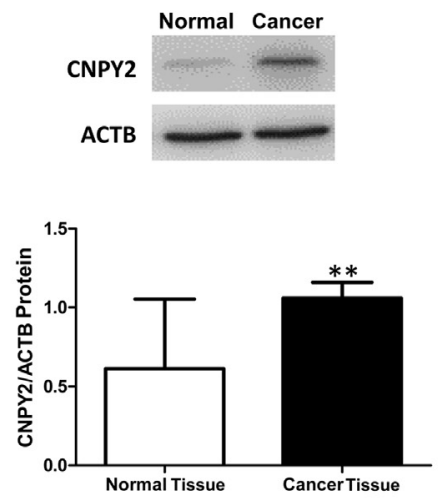

D

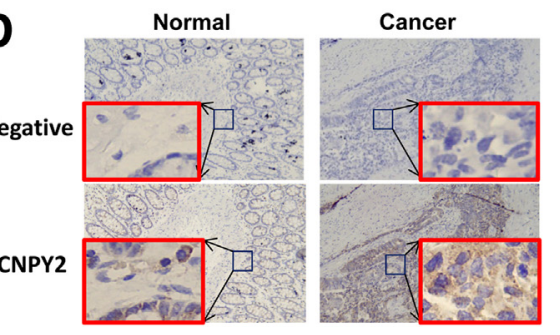

Figure 1 Expression of Canopy 2 (CNPY2) increases in colorectal carcinomic (CRC) tissue and plasma from patients. CNPY2 expression is significantly higher in cancer tissue than in adjacent normal tissue from CRC patients, as evaluated by RT-PCR (A) and Western blot analysis (B). A: A negative control PCR was performed using CDNA from rat heart tissue as a template and human CNPY2-specific primers to exclude non-specific (DNA template) amplification. C: CNPY2 protein levels are significantly higher in plasma from CRC patients compared with healthy controls, as measured by enzyme-linked immunosorbent assay. D: Immunohistochemistry for $\mathrm{CNPY}^{+}$ cells (brown; bottom panels) in cancer tissue and adjacent normal tissue shows that CNPY2 is expressed in gland cells. Antibody specificity is demonstrated by the lack of staining in the negative (isotype control) sections (top panels). ${ }^{*} P<0.01 . n=19$ (A and C, CRC group); $n=28$ (B); $n=11$ (C, healthy controls). Original magnifications: $\times 100$ (D, main images); $\times 400$ (D, insets). ACTB, $\beta$-actin. 
A

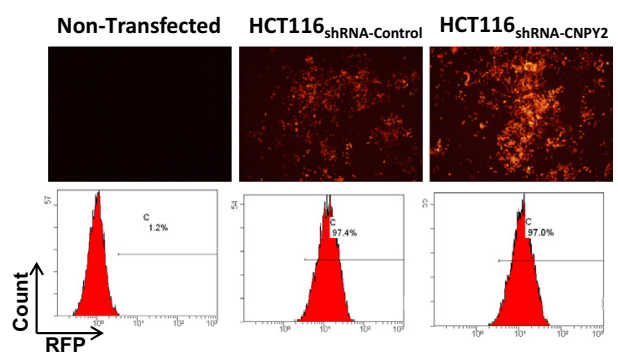

C
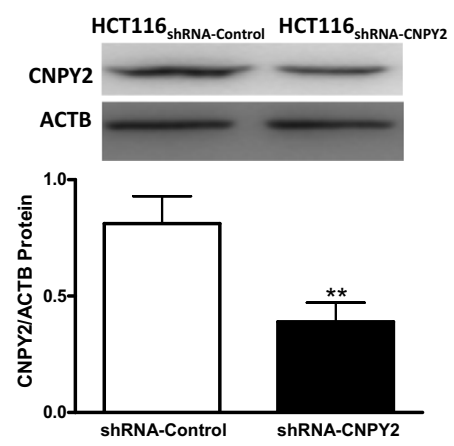
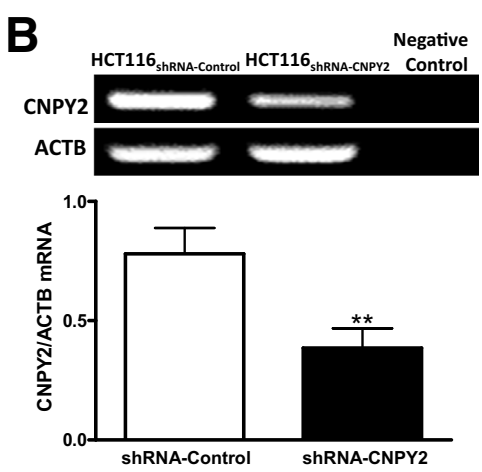

D

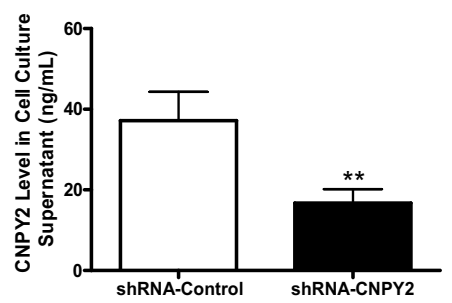

Figure 2 Establishment of stable Canopy 2 (CNPY2) knockdown and control HCT116 human colorectal cancer cell lines. A: Representative micrographs of cells stably transfected with a shRNA targeting CNPY2 (HCT116 $6_{\text {shRNA-CNPY2 }}$ ) and those stably transfected with a control shRNA (HCT116 $\left.6_{\text {shRNA-control }}\right)$. Red staining is cotransfection red fluorescent protein (RFP) marker expression. The establishment of stable cell lines is confirmed by the tagged expression of RFP using flow cytometry analysis. CNPY2 expression is significantly decreased in HCT116 $6_{\text {ShRNA-CNPY2 }}$ cells compared with control HCT116 shRNA-control $_{\text {chells by }}$ RT-PCR (B) and Western blot analysis (C). B: A negative control PCR was performed using CDNA from rat heart tissue as a template and human CNPY2-specific primers to exclude non-specific (DNA template) amplification. D: CNPY2 levels in the supernatant from HCT116 $6_{\text {ShRNA-CNPY2 }}$ cells were significantly decreased compared with control HCT116 $6_{\text {shRNA-control }}$ cells, as measured by enzymelinked immunosorbent assay. ${ }^{*} P<0.01 . n=9$ (B and C); $n=6$ (D). Original magnification, $\times 100$ (A). ACTB, $\beta$-actin. days in culture, the number of colonies per low-power field was analyzed using ImageJ software ( $n=5$ replicates).

\section{Tube Formation Assay}

Human umbilical vein endothelial cells $\left(2 \times 10^{3}\right)$ were cultured with the supernatants derived from HCT116 $6_{\text {shRNA-control }}$ or HCT116 shRNA-CNPY2 $_{\text {in a }}$ 96-well plate, which was precoated with $50 \mu \mathrm{L}$ Matrigel (BD Biosciences, San Jose, CA). After 12 hours, tube formation was observed and recorded using an Olympus microscope (Olympus, Tokyo, Japan). The number of tubes per $\mathrm{mm}^{2}$ was counted and averaged ( $n=5$ replicates).

\section{Cell Apoptosis Assay}

Cells were incubated with 0 to $5 \mu \mathrm{g} / \mathrm{mL}$ oxaliplatin (a tumoricidal drug; JiangSu Hengrui Medicine, Lianyungang, China) for 48 hours and harvested. Apoptosis was tested by flow cytometry (Beckman Coulter) staining for annexin V and propidium iodide (Life Technologies; $n=3$ replicates). For in vivo assessment of apoptosis, terminal deoxynucleotidyl transferasemediated dUTP nick-end labeling (TUNEL) staining (Roche, Basel, Switzerland) was performed following the manufacturer's instruction for the shRNA-control and shRNA-CNPY2 knockdown cell line-induced xenograft tumor tissues.

\section{Tumor Growth and Analysis}

To assess the impact of CNPY2 on tumor cell proliferation in a mouse xenograft model, adult (8 to 10 weeks) male nude mice were injected with HCT116 $6_{\text {shRNA-CNPY2 }}$ and
HCT116 $6_{\text {shRNA-control }}$ cells $\left(3 \times 10^{6}\right)$ s.c. To avoid interaction of the two cell types, shRNA-control or shRNA CNPY2 HCT116 cell lines were injected into separate mice $(n=8)$. To eliminate endogenous factor contribution to tumor growth, we also implanted one cell line under each foreleg of the same mice to generate tumors $(n=10)$. Three weeks after injection, the mice were sacrificed and tumor volume and weight were measured. Tumor volume was calculated using the following formula: $V=A B^{2} / 2$, where $A$ is the largest diameter, and $B$, the smallest. Tumor cells were stained with TUNEL (Roche) to assay apoptosis $(n=3)$.

Immunohistochemistry was conducted using a previously described antigen retrieval protocol, followed by primary antibody incubation. ${ }^{9}$ Rabbit anti-human CNPY2 antibody was used to detect expression of CNPY2 $(n=10)$. Blood vessels were assayed by staining for factor VIII (Abcam, San Francisco, CA) or CD105 (Fujian Maixin Biotechnology, Fujian, China; $n=3$ ), and 5-bromo-2'-deoxyuridine (Abcam) or proliferating cell nuclear antigen staining (Cell Signaling Technology, Danvers, MA; $n=3$ ) was used as a marker for cell proliferation. Imaging and data capture were performed using an upright microscope. Tumor blood vessel density was determined by counting the number of factor $\mathrm{VIII}^{+}$or $\mathrm{CD}_{105^{+}}$vessels per $\times 400$ field. Threshold analysis was performed using ImageJ software.

\section{Caspase- 3 and Caspase- 8 Activity Assays}

Cells $\left(2 \times 10^{6}\right)$ were treated with oxaliplatin $(0,10,20,50$, and $100 \mu \mathrm{g} / \mathrm{mL}$ ) for 48 hours. The activities of caspase- 3 and 
A

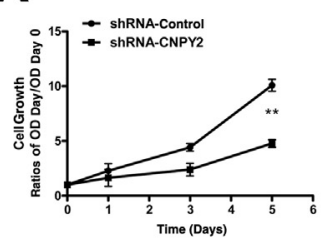

C

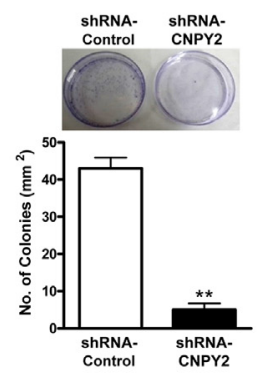

$\mathbf{E}$
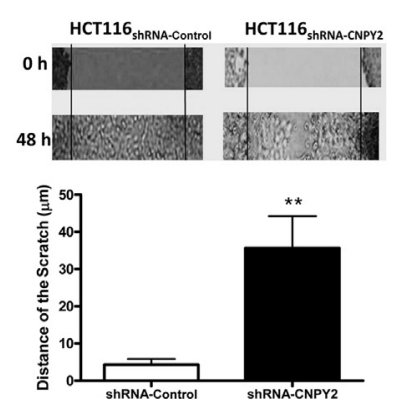

G

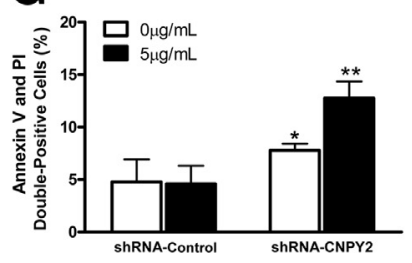

Figure 3 Canopy 2 (CNPY2) knockdown reduces growth, colony formation, migration, and angiogenesis, and increases apoptosis, of human colorectal cancer cells. A: A CCK-8 cell counting assay shows that the number of HCT116 $6_{\text {shRNA-CNPY2 }}$ cells is significantly lower than HCT116 $6_{\text {shRNA-control }}$ cells by days 3 and 5 after seeding with identical numbers of cells. B: The growth of viable cells was assessed on days $1,2,3$, and 5 after seeding with identical numbers of cells. The number of viable cells in HCT116 $6_{\text {ShRNA-CNPY2 }}$ cells is

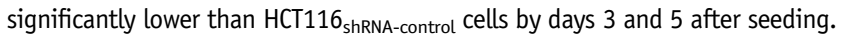
Colony formation with either two-dimensional culture (C) or three-dimensional (3D) soft agar colony assay (D) shows significantly fewer colonies in HCT116 $_{\text {shRNA-CNPY2 }}$ compared with HCT116 shRNA-control $_{\text {(arrows indicate 3D cul- }}$ ture colonies). E: A scratch wound healing migration assay demonstrated that

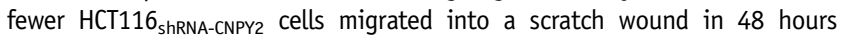
compared with HCT116 shRNA-control $_{\text {cells. F: A Matrigel tube formation assay }}$ demonstrated that fewer tube structures were formed in HCT116 shRNA-CNPY2 $_{\text {cells }}$

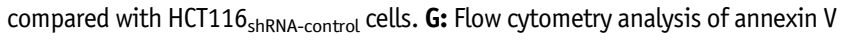
and propidium iodide (PI) double staining as a measure of apoptosis shows that HCT116 $_{\text {ShRNA-CNPY2 }}$ cells have significantly increased apoptosis after 48 hours' incubation with $5 \mu \mathrm{g} / \mathrm{mL}$ oxaliplatin, whereas $\mathrm{HCT}_{116_{\text {shRNA-control }} \text { are resistant }}$ to oxaliplatin-induced apoptosis. ${ }^{*} P<0.05,{ }^{* *} P<0.01 . n=6(\mathbf{A}) ; n=4(\mathbf{B})$; $n=5(\mathbf{C}-\mathbf{F}) ; n=3(\mathbf{G})$. Original magnifications: $\times 40$ (C and $\mathbf{D}$, low-power group); $\times 200$ (C and $\mathbf{D}$, zoomed images). caspase-8 were measured using commercially available kits (Abcam, Cambridge, UK; $n=3$ replicates).

\section{Statistical Analysis}

Data are presented as means \pm SD and analyzed using twotailed unpaired $t$-tests for two-group comparisons and analysis of variance for comparisons of three or more groups. $P<0.05$ was considered statistically significant.

\section{Results}

\section{CNPY2 Expression Increases in CRC Tissues and Plasma from Patients}

Patient characteristics are summarized in Table 1. The expression of CNPY2 in CRC tissue was significantly increased compared with adjacent paracarcinomic tissue $(P<0.01$ for mRNA and protein) (Figure 1, A and B). Moreover, the level of CNPY2 in the plasma of CRC patients was also significantly higher than in healthy controls $(P<0.01)$ (Figure 1C). Immunohistochemistry was used to examine the pattern of expression of CNPY2 in patient tumor samples and showed it was highly expressed in gland cells (Figure 1D). Our results suggest that $C N P Y 2$ may function in CRC.

\section{CNPY2 Knockdown by Stable Transfection of shRNA against CNPY2 in CRC Cells}

To explore the influence of CNPY2 on CRC cells in vitro, we established two stably transfected HCT116 cell lines: one constitutively expressed shRNA-CNPY2 (HCT116 $6_{\text {shRNA-CNPY2) and the other constitutively }}$ expressed a control shRNA (HCT116 $\left.6_{\text {shRNA-control }}\right)$ (Figure 2A). The establishment of stable cell lines was confirmed by the tagged expression of RFP, which was $97.4 \% \pm 0.75 \% \mathrm{RFP}^{+}$in the shRNA control cells and $97 \% \pm 0.83 \% \mathrm{RFP}^{+}$in the shRNA CNPY2 cells, whereas the background non-specific RFP from the non-transfected cells was only $1.2 \% \pm 0.64 \%$ (Figure $2 \mathrm{~A}$ ). We found that HCT116 shRNA-CNPY2 had significantly lower CNPY2 expression by RT-PCR and Western blot analysis $(P<0.01$ for both mRNA and protein) (Figure 2, B and C) and secreted lower levels of CNPY2 into the culture media by enzyme-linked immunosorbent assay $(P<0.01)$ (Figure 2D).

CNPY2 Promotes Growth, Colony Formation, Migration, and Angiogenesis, and Inhibits Apoptosis of HCT116 Cells

Using these cell lines, we observed that cellular growth was significantly inhibited in HCT116 $6_{\text {shRNA-CNPY2 }}$ compared with HCT116 $6_{\text {shRNA-control }}(P<0.01)$ (Figure 3A). This result was confirmed by only assessing viable cell growth and excluding dead cells on days $1,2,3$, and 5 after cell seeding $(P<0.01)$ (Figure 3B). Colony formation with either two-dimensional culture (Figure 3C) or three-dimensional soft agar colony 
A

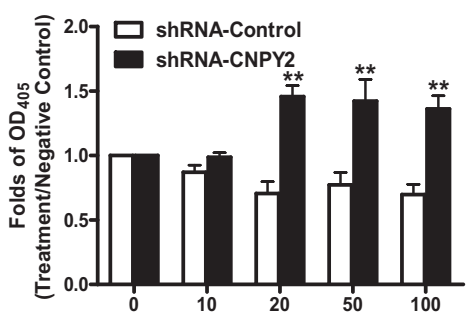

D

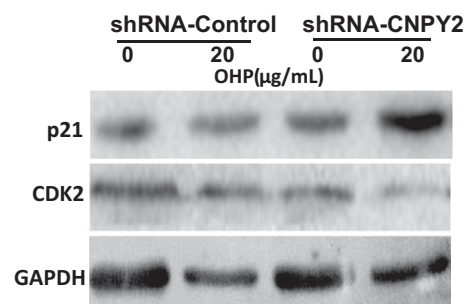

B
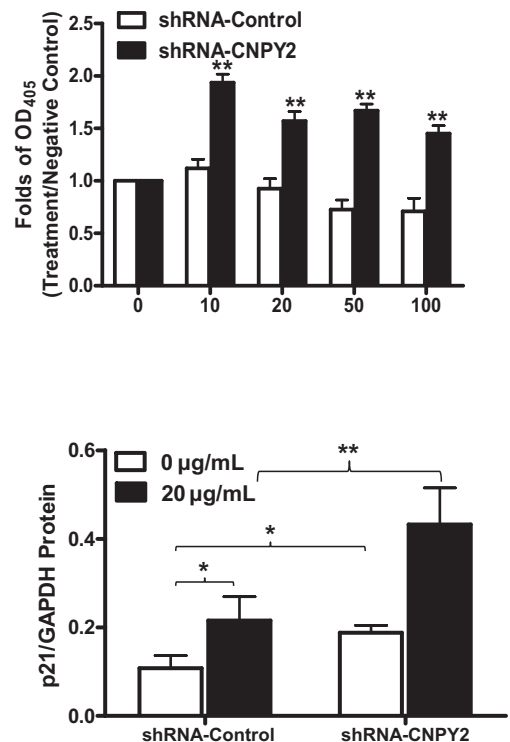

C
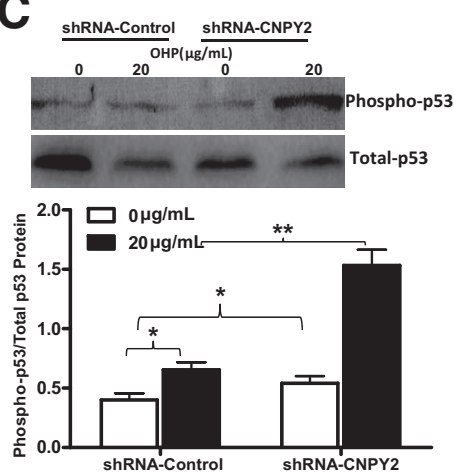

Figure 4 Canopy 2 (CNPY2) knockdown increases oxaliplatin-induced caspase-3 and caspase-8 activities and the ratio of p53 phosphorylated at

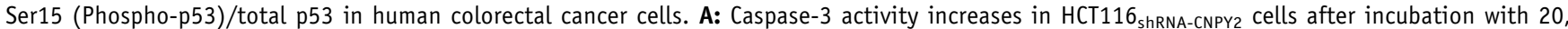

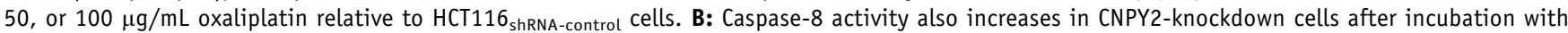
$10,20,50$, and $100 \mu \mathrm{g} / \mathrm{mL}$ oxaliplatin. C: The ratio of phospho-p53/total p53 protein increases in HCT116 $20 \mu \mathrm{g} / \mathrm{mL}$ oxaliplatin (OHP) relative to HCT116 ${ }_{\text {shRNA-control cells. D: Cyclin-dependent kinase (CDK) inhibitor p21 Waf1 }}$ (p21), the downstream mediator

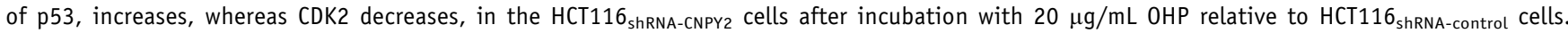
${ }^{*} P<0.05,{ }^{*} P<0.01 . n=3(\mathbf{A}-\mathbf{D})$. GAPDH, glyceraldehyde-3-phosphate dehydrogenase.

assay (Figure 3D) showed significantly fewer colonies in HCT116 $6_{\text {shRNA-CNPY2 compared with HCT116 }}$ shRNA-control ( $P<0.01$ for both assays). Cell migration in a scratch wound healing assay was also significantly decreased in HCT116 shRNA-CNPY2 $_{\text {compared with HCT116 }}$ shRNA-control $(P<0.01)$ (Figure 3E). To investigate in vitro angiogenesis, the Matrigel tube formation assay was used and the number of tubes formed was significantly lower in HCT116 shRNA-CNPY2 $_{2}$ compared with HCT116 shRNA-control $(P<0.05)$ (Figure $3 \mathrm{~F})$. We also explored the role of CNPY2 in oxaliplatin-induced apoptosis in these cell lines. Flow cytometry was used to quantify annexin $\mathrm{V}$ and propidium iodide double staining (both indexes of apoptosis) and showed that apoptosis was significantly enhanced in HCT116 ${ }_{\text {shRNA-CNPY2 }}$ compared with HCT116 $6_{\text {shRNA-control }}$ after 48 hours' incubation with oxaliplatin $(P<0.01)$ (Figure $3 \mathrm{G})$. These results suggest that $C N P Y 2$ enhances the growth and development of CRC by promoting cellular proliferation, migration, and angiogenesis, and inhibiting apoptosis.

\section{Knockdown of CNPY2 Enhances the Apoptosis-} Promoting Activities of Caspase- 3 and Caspase- 8 and Increases p53 Activity

It has been reported that caspase- 3 is activated by both the extrinsic (death ligand) and intrinsic (mitochondrial) apoptotic pathways, whereas caspase- 8 functions only in the extrinsic pathway. ${ }^{10,11}$ To explore the mechanism behind the enhanced oxaliplatin-induced apoptosis in our CNPY2 knockdown cell line, we treated stably transfected HCT116 cells with different concentrations of oxaliplatin for 48 hours. Caspase-3 activity was significantly increased in HCT116 $6_{\text {shRNA-CNPY2 }}$ compared with controls by 20,50 , and $100 \mu \mathrm{g} / \mathrm{mL}$ oxaliplatin $(P<0.01$ for each concentration) (Figure $4 \mathrm{~A}$ ). Caspase- 8 activity was also significantly increased in HCT $116_{\text {shRNA-CNPY2 }}$ after treatment with oxaliplatin compared with HCT116 $6_{\text {shRNA-control }}(P<0.01$ for all concentrations tested) (Figure 4B).

The activity of p53 was evaluated using the ratio of phospho-p53/total p53 proteins. The activity of p53 was increased in HCT116 $6_{\text {ShRA-CNPY2 }}$ cells relative to HCT116 16 shRA-control tumors when the cells were not treated with oxaliplatin $(0 \mu \mathrm{g} / \mathrm{mL}, P<0.05)$ (Figure $4 \mathrm{C}$ ). Expression of p21, the downstream mediator of p53, was increased and CDK2 expression was decreased $(P<0.05)$ (Figure 4D) in HCT116 $6_{\text {shRNA-CNPY2 }}$ cells in comparison with HCT116 16 shNA-control cells. When the cells were treated with $20 \mu \mathrm{g} / \mathrm{mL}$ oxaliplatin, the activation of the p53 pathway (phospho-p53/p53, p21, and CDK2) was further enhanced in HCT116 ${ }_{\text {ShRNA-CNPY2 cells relative to }}$ HCT116 $6_{\text {shRNA-control }}$ cells (Figure 4, C and D). These data 

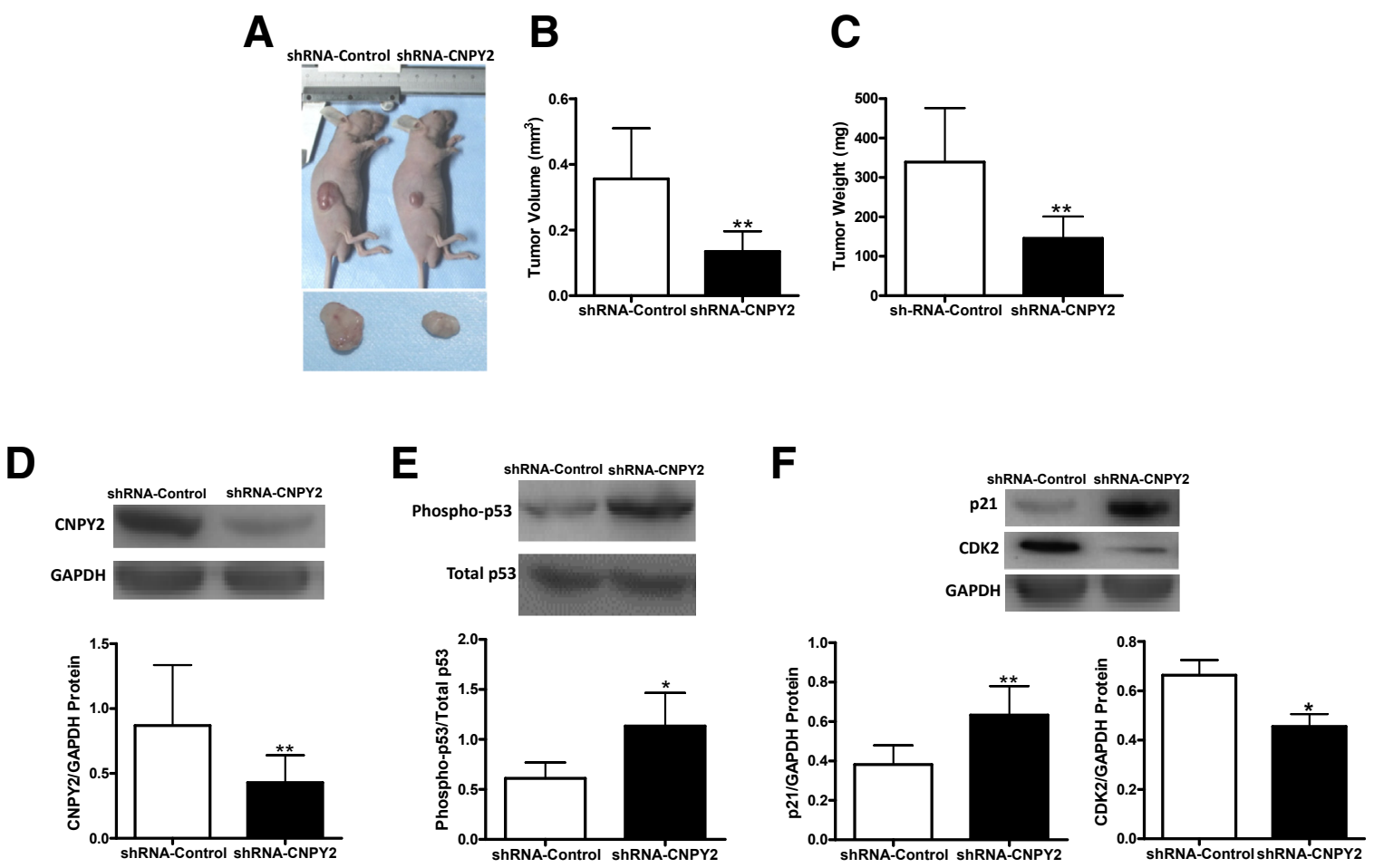

Figure 5 Canopy 2 (CNPY2) knockdown reduces growth of human colorectal tumor (induced in separate mice) and increases the ratio of phospho-p53/total p53. A: HCT116 shRNA-CNPY2 $_{\text {and }}$ HCT116 $6_{\text {ShRNA-control }}$ cells were injected s.c. under the opposite forelegs of nude mice. The xenograft tumors were weighed, measured,

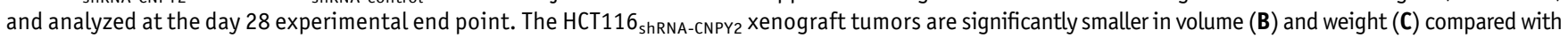
those composed of HCT116 $6_{\text {shRNA-control }}$ cells. D: CNPY2 protein expression significantly reduces in HCT116 $6_{\text {shRNA-CNPY2 }}$ compared to HCT116 shRNA-control tumors. E: The ratio of phospho-p53/total p53 increases in HCT116 shRNA-CNPY2 $_{2}$ compared with HCT116 shRNA-control $_{\text {tumors. F: Cyclin-dependent kinase (CDK) inhibitor p21 }}$ Waf1 $_{\text {(p21) }}$ is up-regulated, whereas CDK2 is down-regulated, in HCT116 shRNA-CNPY2 $_{\text {compared with HCT116 }}$ shRNA-control tumors. ${ }^{*} P<0.05,{ }^{* *} P<0.01 . n=8(\mathbf{B}-\mathbf{D}$ and $\mathbf{F}) ; n=7$ (E). GAPDH, glyceraldehyde-3-phosphate dehydrogenase.

suggest that CNPY2 promotes resistance to antitumor drugs, such as oxaliplatin, by inhibiting the extrinsic and perhaps the intrinsic apoptotic pathways. Cellular apoptosis increased by knockdown of $C N P Y 2$ is associated with the activation of the p53 pathway in HCT116 cells.

\section{CNPY2 Promotes the Growth of HCT116 Tumor Xenografts in Nude Mice in Vivo}

Having found that CNPY2 is highly expressed in CRC tumors and promotes cell proliferation in vitro, we further examined the role of CNPY2 on the growth of CRC cells in vivo. HCT116 $6_{\text {shRNA-control }}$ and HCT116 $6_{\text {shRNA-CNPY2 }}$ cells were injected into nude mice (one cell line under each foreleg) to generate tumor xenografts (Figure 5A and Supplemental Figure S1A). Tumor volume and weight were measured and calculated at the study end point 3 weeks after injection. Both studies showed that tumors derived from HCT116 $6_{\text {shRNA-CNPY2 }}$ were significantly smaller than those from HCT116 $6_{\text {shRNA-control }}$ ( $P<0.05$ and $P<0.01$, respectively, for both measures) (Figure 5, B and C, and Supplemental Figure S1, B and C). CNPY2 protein levels were also significantly lower in the HCT116 $6_{\text {shRNA-CNPY2 }}$ tumors compared with those generated from HCT116 shRNA-control $_{\text {cells }}(P<0.05$ and $P<0.01$, respectively) (Figure 5D and Supplemental Figure S1D).
The ratio of phospho-p53/total p53 proteins was increased in HCT116 $6_{\text {shRNA-CNPY2 }}$ compared with HCT116 shRNA-control $_{\text {sh }}$ derived tumors $(P<0.05)$ (Figure 5E). Consistent with the in vitro results, p21 was up-regulated, whereas CDK2 was down-regulated, in the CNPY2 knockdown cell induced-xenograft tumor tissue compared with the control cell induced-xenograft tumor tissue $(P<0.05$ and $P<0.01$, respectively) (Figure 5F).

We further evaluated cell proliferation, angiogenesis, and apoptosis in these xenografts (Figure 6 and Supplemental Figure S2). We observed that proliferation (assessed by immunohistochemistry against 5-bromo-2'-deoxyuridine and proliferating cell nuclear antigen) and angiogenesis (assayed by factor VIII and CD105 staining) were reduced, and apoptosis (measured by TUNEL staining) was accelerated in the xenografts composed of HCT116 $6_{\text {shRNA-CNPY2 }}$ cells compared with HCT116 $6_{\text {shRNA-control }}(P<0.05$ and $P<0.01$, respectively, for each assay) (Figure 6 and Supplemental Figure S2).

\section{Discussion}

De novo angiogenesis is required to feed tumor growth and is regulated by pro-angiogenic growth and survival factors that are secreted by the malignant cells and other cells 
A

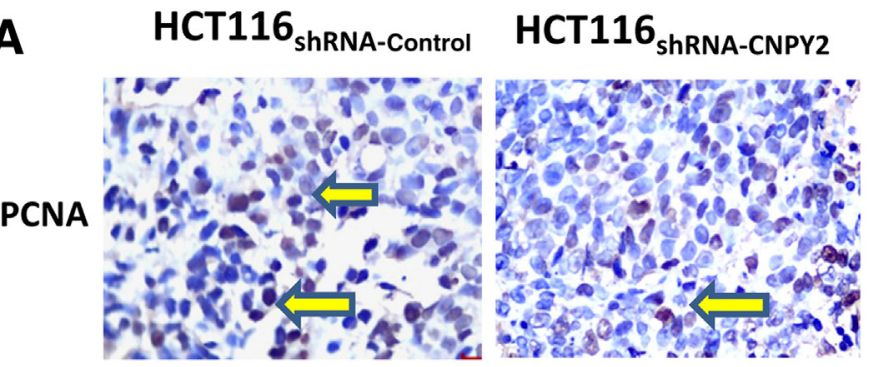

B

BrdU
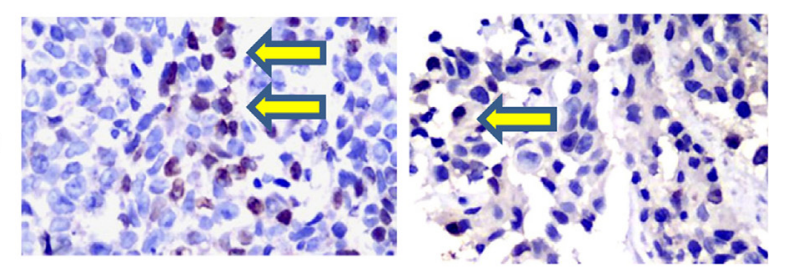

C

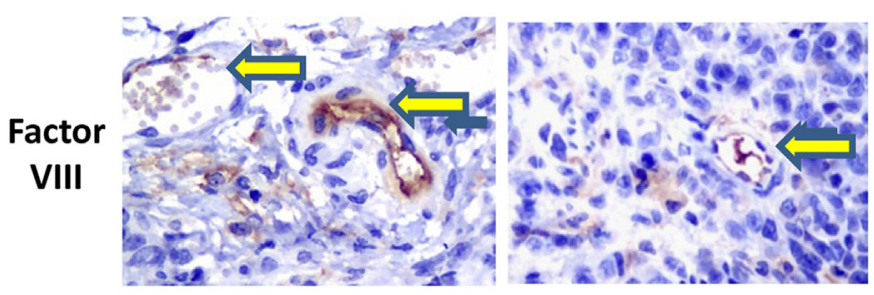

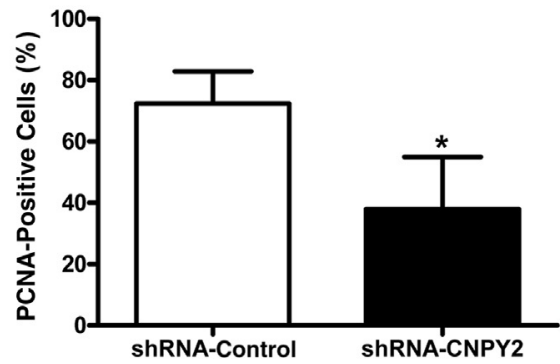
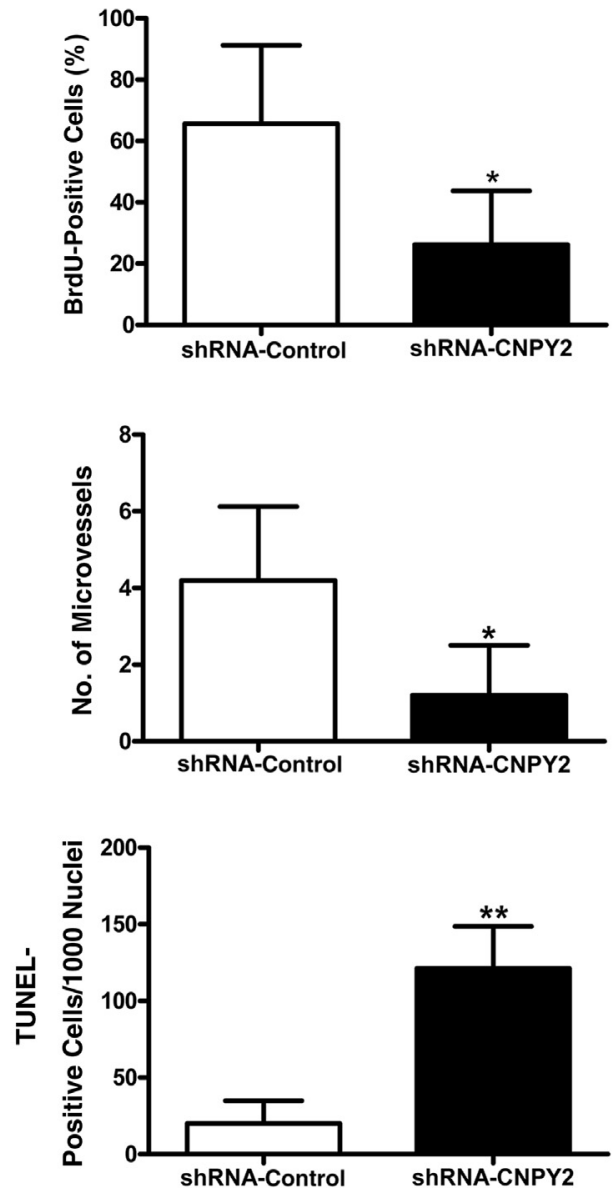

Figure 6 Canopy 2 (CNPY2) knockdown reduces growth, proliferation, and vascularization, and increases apoptosis, of colorectal tumor cells in vivo. A and B: HCT116 $_{\text {ShRNA-CNPY2 }}$ xenograft tumors contain significantly fewer proliferating cell nuclear antigen (PCNA) ${ }^{+}$and 5-bromo-2'-deoxyuridine (BrdU) ${ }^{+}$cells $($brown; arrows) compared with $\mathrm{HCT}_{116_{\text {shRNA-control }}}$ tumors, indicating reduced cellular proliferation. C: HCT116 $_{\text {shRNA-CNPY2 }}$ tumors have significantly reduced angiogenesis [brown factor $\mathrm{VIII}^{+}$staining (arrows)] compared with HCT116 shRNA-control. D: HCT116 shRNA-CNPY2 $_{\text {xenografts have significantly more terminal deoxynucleotidyl }}$ transferase-mediated dUTP nick-end labeling (TUNEL) ${ }^{+}$apoptotic nuclei [dark purple and condensed (arrows)] than HCT116 shRNA-control xenografts. ${ }^{*} P<0.05$, $* * P<0.01 . n=5$ (A-D). Original magnification, $\times 400$ (A-D).

within the tumor microenvironment. Tumor angiogenesis (the ability of a tumor to stimulate new blood vessel formation) is a critical step in tumor development, enabling its expansion, local invasion, and dissemination. ${ }^{4,12-14}$ In a screen for hypoxia-regulated genes that encode secreted proteins, we recently identified a new angiogenic factor, CNPY2, and showed it is a transcriptional target of HIF- $1 \alpha$, is secreted from cells, stimulates smooth muscle cell proliferation and migration, and enhances angiogenesis. ${ }^{6}$ There are only a few other studies that have evaluated the biological function of CNPY2 (alias putative secreted protein Zsig9, transmembrane protein 4, HP10390, and MIRinteracting saposin-like protein), but data support a role for CNPY2 in directed cell growth and migration. When ectopically overexpressed, CNPY2 was reported to enhance neurite outgrowth in neuroblastoma and PC12 cells, and to enhance cell spreading in fibroblasts and migration of rat C6 glioma cells through phosphorylation of myosin regulatory light chain, preventing MIR-mediated myosin regulatory light chain ubiquitination and its subsequent proteasomal degradation. ${ }^{15,16}$ 
In this study, we explored whether this new angiogenic factor was involved in the growth and development of CRC. We found that CNPY2 expression was significantly increased in CRC tissues and in plasma from CRC patients and that CNPY2 could enhance the growth, migration, and angiogenesis, and inhibit the apoptosis, of CRC cells. Consistently, we also showed that $C N P Y 2$ can enhance tumor growth and angiogenesis and reduce apoptosis in vivo.

Angiogenesis is an adaptive response to hypoxia, and HIF$1 \alpha$, with its hypoxia-responsive activation, is a key factor required to induce neovascularization. ${ }^{17}$ Other angiogenic factors are also known to be transcriptionally regulated by HIF-1 $\alpha$, including VEGF. ${ }^{18}$ Preclinical and clinical studies have indicated that VEGF-directed angiogenesis plays an important role in the development of CRC. In preclinical studies, Kondo et $\mathrm{al}^{19}$ found that VEGF-expressing cancers had increased vascularity and metastatic potential than cancers where VEGF was not up-regulated. Clinically, evidence implicates VEGF and tumor angiogenesis as prognostic factors in CRC, with higher VEGF expression being associated with poorer prognosis. ${ }^{20,21}$ Our data showing a role for CNPY2 in CRC growth suggest different angiogenic factors may have independent roles in tumor vascularization, growth, and development. Like VEGF, CNPY2's effect on tumor angiogenesis could play an important role in promoting tumor growth and preventing cell death.

In addition to angiogenesis, other mechanisms might be also involved in the inhibition of tumor growth by CNPY2. In the current study, by knocking down CNPY2 in tumor cells, we found inhibition of tumor cell growth and enhanced apoptosis. P53 is a tumor suppressor and plays a role in cellular apoptosis, genomic stability, and inhibition of angiogenesis. ${ }^{8}$ In the in vitro study, we used shRNA to knock down $C N P Y 2$ gene expression in CRC tumor cells. We found that p53 activity was enhanced in the knockdown cell line in comparison with control. When these cells were implanted into mice to induce xenograft tumor tissue (in vivo study), p53 expression was also elevated in the knockdown cell line induced-xenograft tumor tissue. Furthermore, activation of p53 resulted in the up-regulation of the downstream mediator p21 and down-regulation of CDK2 both in vitro and in vivo. Activating the p53 pathway in CNPY2 knockout cells reduced cell growth, migration, colonial formation, and angiogenesis, but increased cellular apoptosis. In the in vivo study, we also showed that activating the p53 pathway reduced tumor growth, proliferation, and angiogenesis, but increased apoptosis in the knockdown cell-induced xenograft tumor tissue. In summary, we demonstrated that p53 is the central player that connects the various cellular phenotypes associated with the knockdown of CNPY2.

Four decades ago, Folkman ${ }^{22}$ first suggested that antiangiogenic agents represented a potential therapeutic strategy for treating cancer. Anti-VEGF therapy has been explored for treating various cancers both preclinically and clinically. For example, a variety of drugs that target VEGF-A or its receptors effectively prevent the growth of many animal tumors and have demonstrated evidence of antitumor activity in the clinic. ${ }^{23-26}$ However, the remarkable benefits of anti-VEGF/ VEGF receptor therapy observed in animal studies have not fully translated to the clinical setting. These drugs showed only modest effects on human cancers and did not provide the anticipated benefits. For example, an antibody against VEGFA plus triple chemotherapy only prolonged the life of patients with metastatic CRC by 4 to 5 months. ${ }^{27}$ Although anti-VEGF drugs do show some therapeutic efficacy in some kinds of human cancer, the benefits are transient and tumor growth and progression are typically restored. ${ }^{28}$ In addition, recent studies into VEGF inhibitor therapy have shown reductions in the growth of primary tumors but also a concurrent increase in tumor invasiveness and metastasis. ${ }^{29,30}$ Thus, the development of new anti-angiogenic cancer strategies is urgently needed. We propose that CNPY2 could be explored as a new therapeutic target for treating cancers. Our previous work showed that $C N P Y 2$ functions better than VEGF in an ex vivo angiogenic assay. ${ }^{6}$ In addition, we show herein that $C N P Y 2$ can inhibit the extrinsic (death ligand) and possibly the intrinsic (mitochondrial) apoptotic pathways. This novel angiogenic factor may be an attractive anti-cancer therapeutic target, and its effects on angiogenesis and apoptosis in the tumor environment should be investigated further.

\section{Conclusions}

We found that CRC is associated with significantly increased CNPY2 expression. Our study suggests that CNPY2 plays a critical role in the growth and development of CRC by enhancing the proliferation, migration, and angiogenesis, and inhibiting the apoptosis, of CRC cells. We corroborated these in vitro results by showing that $C N P Y 2$ plays a role in tumor growth in vivo. Mechanistically, knockdown of CNPY2 reversibly increases p53 activity, which, in turn, initiates the signal cascade to inhibit tumor cell growth, induce cellular apoptosis, and reduce angiogenesis. Thus, CNPY2 could serve as a novel therapeutic target and prognostic factor for CRC. The mechanism of CNPY2 function within CRC tissues should be investigated further.

\section{Acknowledgment}

We thank Dr. Leigh Botly for assistance with manuscript preparation and editing.

\section{Supplemental Data}

Supplemental material for this article can be found at http://dx.doi.org/10.1016/j.ajpath.2015.11.012.

\section{References}

1. Staib L, Link KH, Blatz A, Beger HG: Surgery of colorectal cancer: surgical morbidity and five- and ten-year results in 2400 
patients: monoinstitutional experience. World J Surg 2002, 26 : 59-66

2. Siegel R, Desantis C, Jemal A: Colorectal cancer statistics, 2014. CA Cancer J Clin 2014, 64:104-117

3. Daly ME, Makris A, Reed M, Lewis CE: Hemostatic regulators of tumor angiogenesis: a source of antiangiogenic agents for cancer treatment? J Natl Cancer Inst 2003, 95:1660-1673

4. Bergers G, Benjamin LE: Tumorigenesis and the angiogenic switch. Nat Rev Cancer 2003, 3:401-410

5. Takahashi Y, Ellis LM, Mai M: The angiogenic switch of human colon cancer occurs simultaneous to initiation of invasion. Oncol Rep 2003, 10: $9-13$

6. Guo J, Zhang Y, Mihic A, Li S-H, Sun Z, Shao Z, Wu J, Weisel RD, Li R-K: A secreted protein (Canopy 2, CNPY2) enhances angiogenesis and promotes smooth muscle cell migration and proliferation. Cardiovasc Res 2015, 105:383-393

7. Sano M, Minamino T, Toko H, Miyauchi H, Orimo M, Qin Y, Akazawa H, Tateno K, Kayama Y, Harada M, Shimizu I, Asahara T, Hamada H, Tomita S, Molkentin JD, Zou Y, Komuro I: P53-induced inhibition of Hif-1 causes cardiac dysfunction during pressure overload. Nature 2007, 446:444-448

8. Levine AJ, Hu W, Feng Z: The P53 pathway: what questions remain to be explored? Cell Death Differ 2006, 13:1027-1036

9. Wang XX, Li XZ, Zhai LQ, Liu ZR, Chen XJ, Pei Y: Overexpression of IQGAP1 in human pancreatic cancer. Hepatobiliary Pancreat Dis Int 2013, 12:540-545

10. Salvesen GS: Caspases: opening the boxes and interpreting the arrows. Cell Death Differ 2002, 9:3-5

11. Ghavami S, Hashemi M, Ande SR, Yeganeh B, Xiao W, Eshraghi M, Bus CJ, Kadkhoda K, Wiechec E, Halayko AJ, Los M: Apoptosis and cancer: mutations within caspase genes. J Med Genet 2009, 46:497-510

12. Hanahan D, Weinberg RA: Hallmarks of cancer: the next generation. Cell 2011, 144:646-674

13. Hicklin DJ, Ellis LM: Role of the vascular endothelial growth factor pathway in tumor growth and angiogenesis. J Clin Oncol 2005, 23: $1011-1027$

14. Nyberg P, Salo T, Kalluri R: Tumor microenvironment and angiogenesis. Front Biosci 2008, 13:6537-6553

15. Bornhauser BC, Lindholm D: MSAP enhances migration of C6 glioma cells through phosphorylation of the myosin regulatory light chain. Cell Mol Life Sci 2005, 62:1260-1266

16. Bornhauser BC, Olsson PA, Lindholm D: MSAP is a novel MIRinteracting protein that enhances neurite outgrowth and increases myosin regulatory light chain. J Biol Chem 2003, 278:35412-35420

17. Pugh CW, Ratcliffe PJ: Regulation of angiogenesis by hypoxia: role of the HIF system. Nat Med 2003, 9:677-684

18. Ahluwalia A, Tarnawski AS: Critical role of hypoxia sensor-HIF-1 $\alpha$ in VEGF gene activation: implications for angiogenesis and tissue injury healing. Curr Med Chem 2012, 19:90-97
19. Kondo Y, Arii S, Mori A, Furutani M, Chiba T, Imamura M: Enhancement of angiogenesis, tumor growth, and metastasis by transfection of vascular endothelial growth factor into LoVo human colon cancer cell line. Clin Cancer Res 2000, 6:622-630

20. Ferroni P, Palmirotta R, Spila A, Martini F, Formica V, Portarena I, Del Monte G, Buonomo O, Roselli M, Guadagni F: Prognostic value of carcinoembryonic antigen and vascular endothelial growth factor tumor tissue content in colorectal cancer. Oncology 2006, 71:176-184

21. Moehler M, Frings C, Mueller A, Gockel I, Schimanski CC, Biesterfeld S, Galle PR, Holtmann MH: VEGF-D expression correlates with colorectal cancer aggressiveness and is downregulated by cetuximab. World J Gastroenterol 2008, 14:4156-4167

22. Folkman J: Tumor angiogenesis: therapeutic implications. N Engl J Med 1971, 285:1182-1186

23. Kim ES, Serur A, Huang J, Manley CA, McCrudden KW, Frischer JS, Soffer SZ, Ring L, New T, Zabski S, Rudge JS, Holash J, Yancopoulos GD, Kandel JJ, Yamashiro DJ: Potent VEGF blockade causes regression of coopted vessels in a model of neuroblastoma. Proc Natl Acad Sci U S A 2002, 99:11399-11404

24. Inai $\mathrm{T}$, Mancuso $\mathrm{M}$, Hashizume $\mathrm{H}$, Baffert $\mathrm{F}$, Haskell $\mathrm{A}$, Baluk $\mathrm{P}, \mathrm{Hu}-$ Lowe DD, Shalinsky DR, Thurston G, Yancopoulos GD, McDonald DM: Inhibition of vascular endothelial growth factor (VEGF) signaling in cancer causes loss of endothelial fenestrations, regression of tumor vessels, and appearance of basement membrane ghosts. Am J Pathol 2004, 165:35-52

25. Carmeliet P, Jain RK: Principles and mechanisms of vessel normalization for cancer and other angiogenic diseases. Nat Rev Drug Discov 2011, 10:417-427

26. Zhu AX, Sahani DV, Duda DG, di Tomaso E, Ancukiewicz M, Catalano OA, Sindhwani V, Blaszkowsky LS, Yoon SS, Lahdenranta J, Bhargava P, Meyerhardt J, Clark JW, Kwak EL, Hezel AF, Miksad R, Abrams TA, Enzinger PC, Fuchs CS, Ryan DP, Jain RK: Efficacy, safety, and potential biomarkers of sunitinib monotherapy in advanced hepatocellular carcinoma: a phase II study. J Clin Oncol 2009, 27:3027-3035

27. Hurwitz H, Fehrenbacher L, Novotny W, Cartwright T, Hainsworth J, Heim W, Berlin J, Baron A, Griffing S, Holmgren E, Ferrara N, Fyfe G, Rogers B, Ross R, Kabbinavar F: Bevacizumab plus irinotecan, fluorouracil, and leucovorin for metastatic colorectal cancer. $\mathrm{N}$ Engl J Med 2004, 350:2335-2342

28. Bergers G, Hanahan D: Modes of resistance to anti-angiogenic therapy. Nat Rev Cancer 2008, 8:592-603

29. Ebos JML, Lee CR, Cruz-Munoz W, Bjarnason GA, Christensen JG, Kerbel RS: Accelerated metastasis after short-term treatment with a potent inhibitor of tumor angiogenesis. Cancer Cell 2009, 15:232-239

30. Pàez-Ribes M, Allen E, Hudock J, Takeda T, Okuyama H, Viñals F, Inoue M, Bergers G, Hanahan D, Casanovas O: Antiangiogenic therapy elicits malignant progression of tumors to increased local invasion and distant metastasis. Cancer Cell 2009, 15:220-231 